\title{
Molecular Size Effect in the Chemical Sputtering of a-C:H Thin Films by Low Energy $\mathrm{H}^{+}, \mathrm{H}_{2}{ }^{+}$, and $\mathrm{H}_{3}{ }^{+}$Ions
}

\author{
P.R. Harris ${ }^{\mathrm{a}, 1}$, F.W. Meyer ${ }^{\mathrm{a}}$, W. Jacob ${ }^{\mathrm{b}}$, T. Schwarz-Selinger ${ }^{\mathrm{b}}$ and U. von Toussaint ${ }^{\mathrm{b}}$ \\ a Physics Division, Oak Ridge National Laboratory, Oak Ridge, Tennessee, 37831-6372, USA \\ ${ }^{b}$ Max-Planck-Institut für Plasmaphysik, EURATOM Association, Boltzmannstr. 2, 85748 \\ Garching, Germany
}

We have experimentally determined total carbon yields per incident $\mathrm{H}$ atom in the energy range 36-300 eV/H for $\mathrm{H}^{+}, \mathrm{H}_{2}{ }^{+}$, and $\mathrm{H}_{3}{ }^{+}$projectiles incident normally on $\sim 60 \mathrm{~nm}$ thick a-C:H films, using 2-D ellipsometry determination of erosion crater volumes exvacuo, the separately characterized thin film carbon density, and the incident beam current integration accumulated on target during the crater evolution. During each beam exposure, methane production was monitored using in-situ quadrupole mass spectrometry (QMS). The present total carbon yields/ $\mathrm{H}$ for incident $\mathrm{H}_{3}{ }^{+}$ions obtained via ellipsometry are in agreement with total mass loss measurements for $\mathrm{H}_{3}{ }^{+}$by Balden and Roth [1] over the investigated energy range. The observed methane production per incident $\mathrm{H}$ for the molecular ions exhibits molecular size effects over the entire energy range investigated, confirming the trend observed in the ellipsometry-based total $\mathrm{C}$ yields/H.

PACS: 34.35.+a; 52.20.Hv; 68.49.Bc; 68.49.Df; 68.49.Sf; 79.20.Rf; 79.20-m;

Keywords: sputtering, ion-surface collisions, chemical erosion, carbon-based materials, divertor materials, a-C:H thin films

\footnotetext{
${ }^{1}$ Corresponding author. e-mail: harrispr@ornl.gov
} 


\section{Introduction}

Plasma facing components (PFC's) of present and future magnetic confinement fusion devices are often comprised of carbon based materials [2]. The response (erosion, desorption of sputtering products) of such PFC's to low energy particle bombardment is strongly influenced by physical and chemical sputtering processes, and has therefore been studied extensively [3]. At low energies, molecular ions have served as a proxy for atomic ions with only limited experimental evidence or theoretical justification $[1,3]$. The commonly made assumption that the sputtering yield per atomic constituent for an incident molecular projectile with energy $E$ is the same as that for an incident $\mathrm{H}^{+}$or $\mathrm{D}^{+}$at energy $E / 2$ and $E / 3$ (for the diatomic and triatomic species, respectively) was examined in the case of methane production by low energy $\mathrm{D}^{+}, \mathrm{D}_{2}{ }^{+}$, and $\mathrm{D}_{3}{ }^{+}$ions incident on ATJ graphite (UCAR Carbon Co.) in [4], and has recently been tested for total carbon yields in the sputtering of a-C:D by atomic and molecular deuterium ions in [5]. In the latter study it was found that total carbon yields per incident $\mathrm{D}$ for incident $\mathrm{D}_{3}{ }^{+}$ions were a factor of 2-3 larger than the corresponding yields for $\mathrm{D}^{+}$ions for incident energies in the range $30-80 \mathrm{eV} / \mathrm{D}$. At $200 \mathrm{eV} / \mathrm{D}$ the differences in total carbon sputtering yields/D for $\mathrm{D}^{+}, \mathrm{D}_{2}{ }^{+}$, and $\mathrm{D}_{3}{ }^{+}$incident ions were within the experimental uncertainty [5]. In addition to such experimental evidence, differences in total carbon erosion induced by atomic and molecular projectiles by factors exceeding the projectile atomic constituency number were also found in molecular dynamics simulations $[4,6]$. For clarity, when comparing same velocity atomic and molecular ions, we refer to yield enhancements by factors exceeding the projectile atomic constituency number as exhibiting molecular size effects. In the present article, we have investigated the molecular size effects arising in the sputtering of a-C:H thin films by hydrogen atomic and molecular ion projectiles incident in the energy range 36-300 eV/H, using an in-situ QMS approach for methane yields, and 2-D ex-vacuo ellipsometry for total carbon yields.

In view of the trend towards lower divertor temperatures and resulting higher plasma abundances of molecular ions, molecular size effects in chemical sputtering have important implications for plasma wall interactions, both for lifetimes of C-based PFC's and for $\mathrm{H}, \mathrm{D}, \mathrm{T}$ retention. To facilitate assessment for heavier $\mathrm{H}$ isotopes the present measurements and those of [5] are compared with recent molecular dynamics calculations by Reinhold et al. [7].

\section{Experimental Procedure}

The experimental apparatus employed in this work has been previously described $[8,9,10]$. All measurements were made in a floating potential, ultra-high vacuum, target chamber with base pressures in the $10^{-8} \mathrm{~Pa}$ range, into which $10 \mathrm{keV}$ beams extracted from an electron cyclotron resonance (ECR) source were delivered and decelerated [11]. The decelerated beams were well defined spatially, as determined by a wire scanner measuring beam current profiles in the sample plane, and energetically, as determined by measurements of the target beam current as a function of chamber bias voltage [12]. Impact energies of the decelerated incident projectiles were determined from the relative bias between the ECR ion source voltage and the target chamber, and corrected for the finite ECR source plasma potential of $+14 \mathrm{~V}$ [12] and for the $+28 \mathrm{~V}$ sample bias to 
suppress secondary electron emission, when employed. An estimate of the beam flux incident on the sample was obtained from the measured sample beam current and the 1-D spatial current distribution measured with the movable wire scanner. Fluxes exceeding 1 $\mathrm{x} 10^{15} \mathrm{H} \mathrm{cm}^{-2}-\mathrm{s}^{-1}$ were obtained at all the energies investigated. All the measurements were carried out under normal incidence conditions, and for room temperature targets.

In the present measurements, plasma-deposited amorphous hydrogenated carbon thin films $(\mathrm{a}-\mathrm{C}: \mathrm{H})$ were used to serve as a model system for hydrocarbon surfaces found in magnetic confinement fusion devices. a-C:H films were deposited on $400 \mu \mathrm{m}$ thick, polished single crystalline silicon wafers $(\mathrm{Si}(001))$ mounted at the driven electrode of an asymmetrical, capacitively coupled discharge $(13.56 \mathrm{MHz})$. Film growth was monitored in-situ with interferometry. Pure $\mathrm{CH}_{4}$ was used for the film deposition at a gas pressure of $2 \mathrm{~Pa}$ and a total $\mathrm{CH}_{4}$ gas flow of 20 standard cubic centimeters per minute $(\mathrm{sccm})$. The applied rf power of $26 \mathrm{~W}$ resulted in a dc self-bias voltage of $-300 \mathrm{~V}$. Properties of a-C:H films deposited in a similar fashion have been described by Schwarz-Selinger et al. [13]. With the $\mathrm{CH}_{4}$ feed gas, films with a hydrogen content $(\mathrm{H} /(\mathrm{H}+\mathrm{C}))$ of $30 \%$, refractive index of $\mathrm{n}=2.13-0.12 \mathrm{i}$ at $632.8 \mathrm{~nm}$ and a carbon number density of $(8.5 \pm 0.2) \times 10^{22} \mathrm{~cm}^{-3}$ are produced. To obtain close to identical properties for each erosion measurement, films were initially deposited on $10 \mathrm{~cm}$ diameter wafers that were subsequently hand cut into pieces $25 \mathrm{~mm} \times 25 \mathrm{~mm}$ in size.

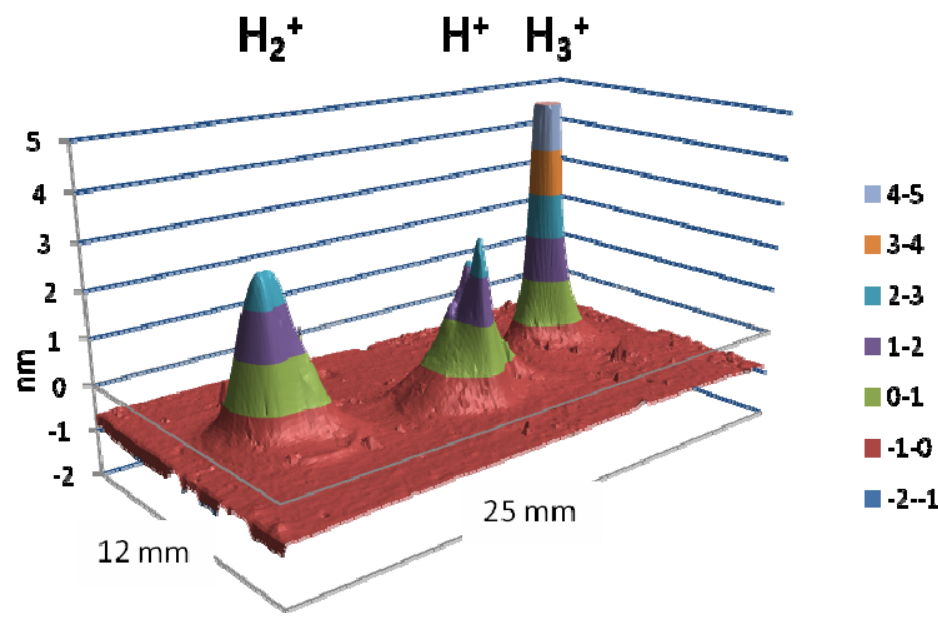

Figure 1: Difference of 2-D ellipsometry post- and pre- exposure scans after irradiation by three different ion beams (from left to right, $\mathrm{H}_{2}{ }^{+}, \mathrm{H}^{+}$, and $\mathrm{H}_{3}{ }^{+}$, all at $36 \mathrm{eV} / \mathrm{H}$ ) incident on different regions of an a-C:H target sample, showing typical crater depth dimensions. Note that horizontal dimensions are in $\mathrm{mm}$, while the vertical dimension is in $\mathrm{nm}$.

The film thickness distributions on each sample were measured before and after exposure to the ion beams with ex-situ 2-D ellipsometry. Details of this procedure are described more fully in [5]. Differences between pre and post beam exposure scans were taken to determine spatially resolved net thickness changes of the thin films resulting from the beam exposures. This eliminated the effects of slight thickness non-uniformities, introduced during the film deposition process, on the crater volume determination. Figure 1 shows a typical post/pre-exposure subtraction, and illustrates the isolated craters 
produced by three different ion beams superimposed on a homogenous flat background. Note that in Figure 1 the crater diameters are six orders of magnitude larger than the crater depths ( $\mathrm{mm}$ vs. $\mathrm{nm}$ ) The negative 0.6-0.8 $\mathrm{nm}$ offset evident in the unexposed regions of the thin film target is attributed [5] to a slight relaxation of the a-C:H films which presumably occurred during the 4 day $100^{\circ} \mathrm{C}$ chamber bake carried out prior to the beam exposures. The effect of the resulting uniform swelling on a reduction of the $\mathrm{C}$ film density amounted to less than $2 \%$ and was neglected in the subsequent analysis. Before crater volumes were calculated, isolated outlier pixels were removed using a 5 x 5 pixel-median filter applied to the height-fields of the unexposed and exposed sample. The crater area was determined from the product of the known pixel area and the number of pixels in each crater, while the crater volume was determined by summing the crater depths measured for each crater pixel. The total eroded carbon amount of the individual crater was determined by converting the integrated crater volume using the carbon density quoted above.

As already indicated above, the a-C:H thin film samples could be biased at $+28 \mathrm{~V}$ when required, to suppress emission of secondary electrons produced by ion beam impact at the higher investigated beam energies. Such sample biasing was used at the two highest investigated energies of $140 \mathrm{eV} / \mathrm{H}$ and $300 \mathrm{eV} / \mathrm{H}$. By comparing sample currents with and without biasing, secondary electron coefficients of $0.028,0.045,0.069$ were determined for incident $140 \mathrm{eV} / \mathrm{H} \mathrm{H}^{+}, \mathrm{H}_{2}{ }^{+}, \mathrm{H}_{3}{ }^{+}$ions, respectively. Based on this measurement and published results for secondary electron emission coefficients for $\mathrm{D}$ atomic and molecular ion beams incident on highly ordered pyrolytic graphite (HOPG) [14], $+28 \mathrm{~V}$ sample biasing was only used for ion energies at $140 \mathrm{eV} / \mathrm{H}$ or higher. At the lower investigated energies, secondary electron emission was assumed to be negligibly small compared to the other experimental uncertainties, and the positive sample bias was not applied.

All $\mathrm{H}$ beam exposures were carried out to fluences of at least $10^{19} \mathrm{H} \mathrm{cm}^{-2}$. By reaching such fluences, it was ensured that the majority of erosion occurred under steadystate sputtering conditions. Such fluences were well beyond the $10^{18} \mathrm{D} \mathrm{cm}^{-2}$ required to reach steady-state $\mathrm{CD}_{4}$ production in ATJ graphite [9] from zero initial D content. Since the a-C:H thin films were pre-hydrogenated to $\sim 30 \%$, it is expected that steady-state conditions were reached at fluences below $10^{18} \mathrm{H} \mathrm{cm}^{-2}$. In the present measurements, attainment of steady-state conditions in hydrocarbon production was monitored by periodic QMS scans during beam dosing, with typically 3-5 full-mass-range spectra being acquired over a 30-90 minute time span. Acquisition of such full-mass-range (1-75 $\mathrm{amu} / \mathrm{q}$ ) spectra was augmented by measurements of transient spectra for selected hydrocarbons at beam turn-on and turn-off.

In addition to the net erosion yields determined from the ellipsometry measurements, we focus in this paper on the analysis of methane production via QMS based on transient measurements (i.e. time-base scans) taken when the beam was first turned on and at the end of each beam exposure, when steady-state conditions had been attained [10], as well as on full-range mass spectra close to the end of a beam exposure cycle. In all cases the final transient measurements either confirmed or exceeded the molecular size effects already evident in the initial transients. To reduce fluctuations in the transients due to low frequency noise in the analog output of our ageing QMS, all transients were smoothed using a 15 channel running sum average. For the methane 
production, we ignored the contribution of heavier hydrocarbons at the methane analysis mass $=15$, since the cracking patterns of heavier hydrocarbons contribute only a small percentage to the $m=15$ intensity.

\section{Results}

Figure 2 shows total carbon chemical sputtering yields per $\mathrm{H}$ determined via 2-D ellipsometry measurements for $\mathrm{H}^{+}, \mathrm{H}_{2}{ }^{+}$, and $\mathrm{H}_{3}{ }^{+}$beams incident on a-C: $\mathrm{H}$ thin films in the energy range $36-300 \mathrm{eV} / \mathrm{H}$. The results are summarized in Table I. As can be seen in Figure 2, the present ellipsometry-based results for $\mathrm{H}_{3}{ }^{+}$are in good agreement with the mass loss results of [1] for the same projectile. At $200 \mathrm{eV} / \mathrm{H}$, the $\mathrm{H}$ ion projectiles were incident on an a-C:D thin film, prepared as described in [5], rather than the a-C:H films used for the other investigated energies. As expected, the initial condition of the thin film (deuterated rather than hydrogenated) did not significantly affect the steady-state yields. Due to the hydrogenation and isotope exchange reactions occurring during the cumulative $\mathrm{H}$ ion bombardment, the near-surface composition of the a-C:D thin film at steady state is expected to be very similar to that of the steady-state a-C:H thin films, and the yields measured for the a-C:D film at $200 \mathrm{eV} / \mathrm{H}$ are consistent with the trends in energy and incident projectile established by the other a-C:H film measurements. At all seven investigated energies, the yields per $\mathrm{H}_{\text {for }} \mathrm{H}_{3}{ }^{+}$are larger than those for $\mathrm{H}_{2}{ }^{+}$and $\mathrm{H}^{+}$, indicating a significant molecular size effect. Table I makes clear that, with the exception of $200 \mathrm{eV} / \mathrm{H}$ and $300 \mathrm{eV} / \mathrm{H}$ - the highest energies investigated, the $\mathrm{H}_{3}{ }^{+}$and $\mathrm{H}^{+}$yields per $\mathrm{H}$ differ by more than the combined experimental uncertainties.

Table I - Total carbon sputtering yields in units of $\mathrm{C} / \mathrm{H}$ for $\mathrm{H}^{+}, \mathrm{H}_{2}{ }^{+}$, and $\mathrm{H}_{3}{ }^{+}$ions incident on room temperature a-C:H thin films; total uncertainty contains 5\% systematic uncertainty in $C$ density determination and $5 \%$ in ion fluence determination, added in quadrature with the uncertainties in the individual crater volume determinations.

\begin{tabular}{llll}
\hline Energy $(\mathrm{eV} / \mathrm{H})$ & Ion & \\
& $\mathrm{H}^{+}$ & $\mathrm{H}_{2}{ }^{+}$ & $\mathrm{H}_{3}{ }^{+}$ \\
\hline 36 & $0.0025 \pm 0.0005$ & $0.0037 \pm 0.0007$ & $0.0043 \pm 0.0008$ \\
60 & $0.0016 \pm 0.0003$ & $0.0025 \pm 0.0005$ & $0.0038 \pm 0.0008$ \\
72 & $0.0008 \pm 0.0003$ & $0.0020 \pm 0.0004$ & $0.0029 \pm 0.0006$ \\
100 & $0.0015 \pm 0.0003$ & $0.0025 \pm 0.0005$ & $0.0033 \pm 0.0007$ \\
140 & $0.0027 \pm 0.0005$ & $0.0030 \pm 0.0006$ & $0.0055 \pm 0.0010$ \\
200 & $0.0043 \pm 0.0009$ & $0.0058 \pm 0.0011$ & $0.0068 \pm 0.0015$ \\
300 & $0.0049 \pm 0.0014$ & $0.0031 \pm 0.0011$ & $0.0066 \pm 0.0024$ \\
\hline
\end{tabular}




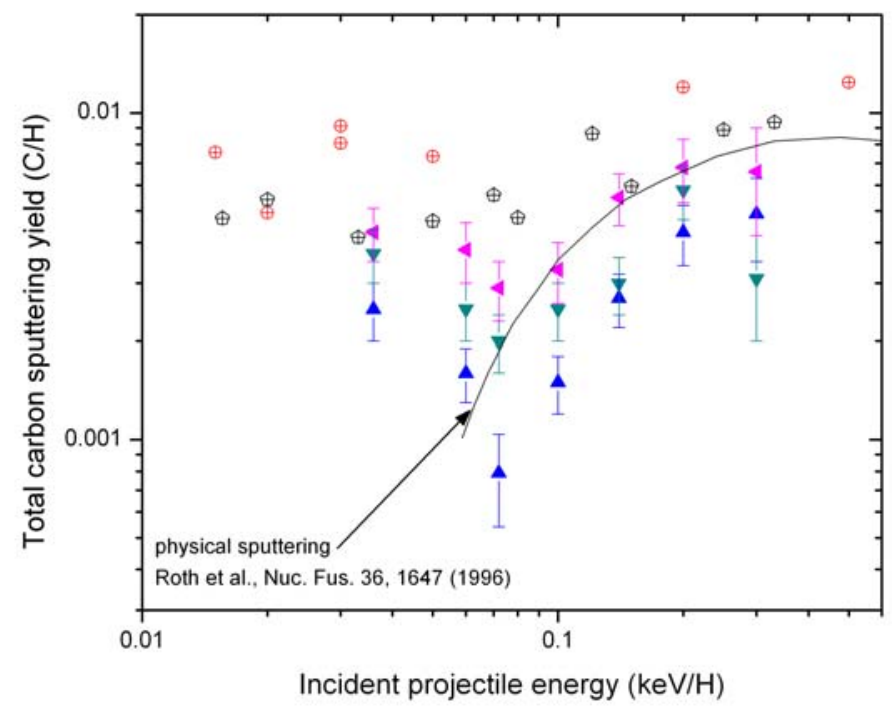

Figure 2: Total C erosion yields in units of carbon atoms per $\mathrm{H}$ for $\mathrm{H}^{+}$(upward triangles), $\mathrm{H}_{2}{ }^{+}$(downward triangles), and $\mathrm{H}_{3}{ }^{+}$(leftward triangles) ions incident on room temperature $a-C: H$ thin films as a function of the energy/ $H$; round and pentagon crosses: total mass loss results of [1] for $\mathrm{H}_{3}{ }^{+}$incident on room temperature pyrolytic graphite along with other $C$ materials. Also shown is the contribution of physical sputtering at the higher energies, as presented in [1].

The methane production $\left(\mathrm{CH}_{4} / \mathrm{H}\right)$ measured in conjunction with these total erosion determinations, is summarized in Figure 3. The observed methane signal for each incident beam was first normalized by the number per second of incident $\mathrm{H}$ atoms in the respective $\mathrm{H}^{+}, \mathrm{H}_{2}{ }^{+}$, and $\mathrm{H}_{3}{ }^{+}$beams. The $\mathrm{H}$-atom-normalized methane production for incident $\mathrm{H}_{2}{ }^{+}$and incident $\mathrm{H}_{3}{ }^{+}$, respectively, was then divided by the corresponding methane production for incident $\mathrm{H}^{+}$, to determine the magnitude of the molecular size effect at each investigated energy. The methane signal was determined from the transient QMS spectra taken at beam turn-off, and/or from one of the full-mass-range scans taken close to the end of a given beam exposure. The presence of molecular size effects is already apparent in transient QMS spectra at beam turn-on, as shown in Figure 4, and is thus not a feature that only appears with increasing fluence. For the full-mass-range spectra, the magnitude of the methane signal was determined two different ways: from the area of the entire mass- 15 peak, or from the mass- 15 peak height alone. Where available, the methane molecular size effects shown in Figure 3 are averages over all three methane determination methods, i.e. the mass- 15 transient spectra level, and the area and peak height in the full-mass-range spectra. In the case of a few data sets, saved files of end transient runs were corrupted (likely due to the sparking affecting the data acquisition computer which was floating at $\sim 10 \mathrm{kV}$ ), and could not be used. In those cases we determined the methane production just from one of the full-mass-range scans taken close to the end of a given beam exposure. Due to the initial $\sim 30 \% \mathrm{H}$ inventory in the a-C:H thin films, the measured methane production could not be corrected for wall collisions. 


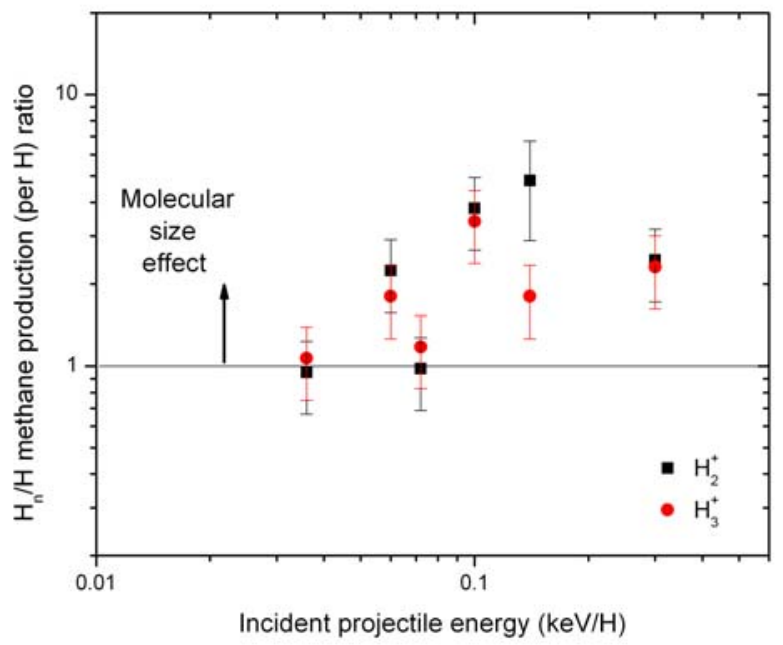

Figure 3: Molecular size effect in methane production by $\mathrm{H}_{2}{ }^{+}$and $\mathrm{H}_{3}{ }^{+}$ions as function of the beam energy/H. Note all $\mathrm{H}_{n}^{+} / \mathrm{H}^{+}$methane production (per $\mathrm{H}$ ) ratios greater than 1 signify a molecular size effect (see text).

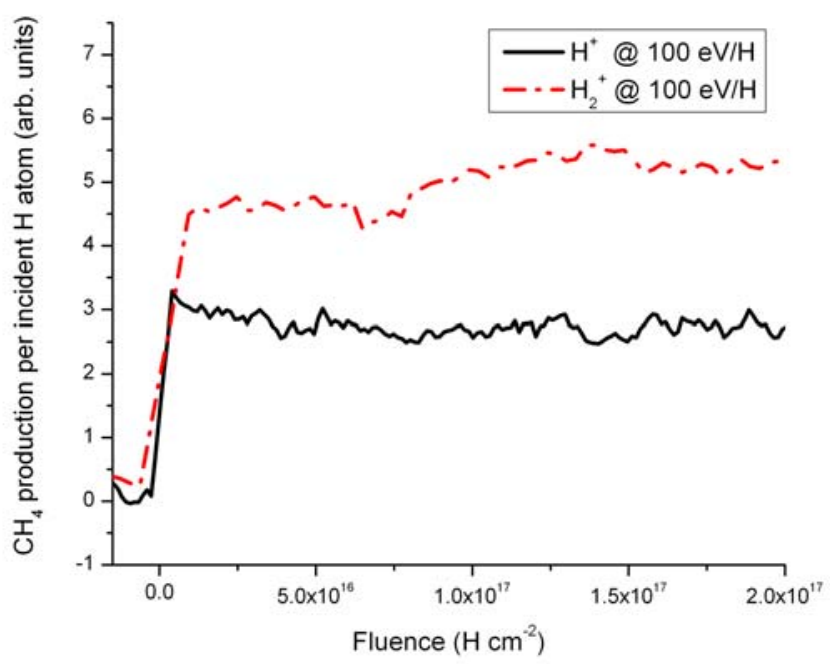

Figure 4: Mass-15 transient spectra acquired at beam turn-on, normalized to the respective $\mathrm{H}$ particle currents, for $\mathrm{H}^{+}$and $\mathrm{H}_{2}{ }^{+}$projectiles incident on a room temperature $a-C: H$ thin film target at $100 \mathrm{eV} / \mathrm{H}$, as a function of $\mathrm{H}$ fluence. Note that the molecular size effect is already evident at beam turn-on. The transients have been subtracted by two (different) beam-off background levels, which were determined outside the abscissa range shown in the figure. 


\section{Discussion}

As the chemical sputtering yields presented here (both for total $\mathrm{C}$ and methane) are quoted per number of incident $\mathrm{H}$ atoms (i.e., already divided by 2 or 3 for incident $\mathrm{H}_{2}{ }^{+}$or $\mathrm{H}_{3}{ }^{+}$projectiles, respectively), the observed molecular size effects for molecular ion projectiles at energies ranging from $36-300 \mathrm{eV} / \mathrm{H}$ are significant. Averaging the molecular size effect enhancement ratios (ratio of the $\mathrm{H}$ normalized molecular projectile induced methane yield to the atomic projectile induced methane yield, e.g., Figure 3) across all investigate energies we find enhancement ratios of $2.54 \pm 0.63$ and $1.93 \pm 0.35$ (at $68 \%$ confidence level), for $\mathrm{H}_{2}{ }^{+}$and $\mathrm{H}_{3}{ }^{+}$, respectively. This is consistent (within the standard deviations of the sample) with the observed average molecular size effect enhancement ratios for total carbon yields of $1.47 \pm 0.22$ and $2.13 \pm 0.28$ (at $68 \%$ confidence level), for $\mathrm{H}_{2}{ }^{+}$and $\mathrm{H}_{3}{ }^{+}$, respectively. These ratios make clear, that even after normalizing yields to the $\mathrm{H}$ atom count, we observe significant molecular size effects for molecular ion projectiles consisting of yields (per $\mathrm{H}$ ) $\sim 2$ larger than those from their atomic ion projectile counterparts.

For the total carbon yields, the enhancements are most prevalent at energies of $200 \mathrm{eV} / \mathrm{H}$ and below, suggesting that at such energies, molecular ions remain intact or produce dissociation fragments that are still spatially correlated for a substantial portion of their range within the a-C:H thin film. Previous work [15] investigating differences in surface damage (studied by use of a scanning Raman microprobe) induced by same velocity atomic and molecular projectiles and compared at similar $\mathrm{H}$ atom fluences supports this notion. In the latter work, increased ATJ graphite surface damage was found for $\mathrm{D}_{3}{ }^{+}$projectiles as compared to same velocity, same fluence D projectiles. Intact molecules or highly spatially correlated fragments have a higher energy deposition density than the corresponding same velocity atomic ions and may thus result in more efficient kinetic ejection of hydrocarbon precursors formed by the cumulative $\mathrm{H}$ ion bombardment. At elevated sample temperatures, where thermally activated release becomes significant and the importance of kinetic ejection processes is correspondingly reduced, one might therefore expect a reduced yield enhancement due to molecular size effects. Future studies are planned to investigate this important issue.

As discussed in previous work on D ions [5], in contrast to the present measurements, the assumption in [1] that molecular ion projectiles are a suitable proxy at low energies for atomic ion projectiles was checked at only one energy $(60 \mathrm{eV} / \mathrm{D})$. At that energy, the $\mathrm{D}^{+}$induced yield was found to be somewhat smaller than that for $\mathrm{D}_{3}{ }^{+}$, however not significantly so compared to the large scatter in the repeated measurements for $\mathrm{D}_{3}{ }^{+}$at that energy, and thus not definitive with respect to the existence of a molecular size effect.

The significant scatter evident in Figure 3 is due in part to the inability to make proper wall collision corrections [8] for the already hydrogenated thin films. Such wall corrections depend directly on projectile energy and thus can be different for the various projectiles plotted at a fixed velocity. Wall corrections were found to increase with increasing energy above $100 \mathrm{eV} / \mathrm{H}$ (see, e.g. Figure 2 in [10]), where the chemical sputtering signal decreases with increasing energy much faster than the wall correction background. Future work using a line-of-sight QMS system is planned, which will be less 
sensitive to wall collision effects, and which will thus allow a more rigorous quantitative evaluation of the fractional contribution of sputtered methane to the total $\mathrm{C}$ production.

In previous measurements of methane production by $\mathrm{H}$ and $\mathrm{D}$ ion bombardment on ATJ graphite $[4,16]$, where the investigated energies ranged from $10-300 \mathrm{eV} / \mathrm{H}$ and $10-60 \mathrm{eV} / \mathrm{D}$, respectively, the molecular size effect was most prominent at the lowest investigated energies, with the effect becoming less significant as energy increased. In contrast, for the present results on a-C:H, the molecular size effect is not limited to the lowest investigated energies. It is not clear at present whether these different trends are due to the different targets (ATJ graphite vs. a-C:H thin film), to a possible isotope effect in the molecular enhancements, or to other variations inherent in the experiment.

As a concluding observation, when compared to previous results for $\mathrm{D}$ projectiles [5], the present ellipsometry results for $\mathrm{H}$ beams permit an estimate to be made of the projectile isotope effect in chemical sputtering. Focusing on the ellipsometry data for energies below $\sim 100 \mathrm{eV} / \mathrm{H}$ or $\mathrm{D}$, i.e. at or below the physical sputtering threshold where chemical sputtering dominates, we see an average difference between $\mathrm{H}^{+}$and $\mathrm{D}^{+}$induced total $\mathrm{C}$ yields of a factor in the range 8-11. Recently published MD simulations comparing such species found an isotope effect magnitude in the total $\mathrm{C}$ production yields closer to a factor of $\sim 2-3$ [7], although the simulations were restricted to energies below $20 \mathrm{eV} / \mathrm{D}$ or $\mathrm{H}$, i.e. well below the present experimental energy range. Since the mass loss results of [1] show an $\mathrm{D} / \mathrm{H}$ isotope effect at low energies closer to our own results (in the range 6-9), either the simulation results systematically underestimate the actual isotope effect magnitude, or the isotope effect magnitude increases strongly with increasing energies above $20 \mathrm{eV}$.

\section{Acknowledgments}

This work was supported by the Office of Fusion Energy Sciences and the Division of Chemical Sciences, Geosciences, and Biosciences, Office of Basic Energy Sciences of the U.S. Department of Energy. PRH was appointed through the ORNL Postdoctoral Research Associates Program administered jointly by Oak Ridge Institute for Science and Education and Oak Ridge National Laboratory. 


\section{References}

[1] M. Balden, J. Roth, J. Nucl. Mater. 280, 39 (2000).

[2] G. Federici, Phys. Scr. T124, 1 (2006).

[3] W. Jacob, J. Roth, in: R. Behrisch, W. Eckstein (Eds.), Sputtering by Particle Bombardment: Experiments and Computer Simulations from Threshold to $\mathrm{MeV}$, Springer, Berlin, 329-400 (2007).

[4] L.I. Vergara, F.W. Meyer, H.F. Krause, P. Träskelin, K. Nordlund, amd E. Salonen, J. Nucl. Mat. 357, 9 (2006).

[5] F.W. Meyer, P.R. Harris, H. Zhang, W. Jacob, T. Schwarz-Selinger, U. von Toussaint, J. Nucl. Mater. (2010) doi: 10.1016/j.jnucmat.2010.08.037.

[6] P.S. Krstic, C.O. Reinhold, S.J. Stuart, Europhys. Lett. 77, 33002 (2007).

[7] C.O. Reinhold, P.S. Krstic, S.J. Stuart, H. Zhang, P.R. Harris, F.W. Meyer, J. Nucl. Mater 401, 1 (2010).

[8] L.I. Vergara, F.W. Meyer, H.F. Krause, J. Nucl. Mater. 347, 118 (2005).

[9] F.W. Meyer, H. Zhang, L.I. Vergara, H.F. Krause, Nucl. Instr. Meth. Phys. Res. B 258, 264 (2007).

[10] H. Zhang and F.W. Meyer, J. Nucl. Mat. 390, 127 (2009).

[11] V.A. Morozov, F.W. Meyer, Rev. Sci. Instrum. 70, 4515 (1999).

[12] P.R. Harris, F.W. Meyer, Rev. Sci. Instrum. 81, 02A310 (2010).

[13] T. Schwarz-Selinger, A. von Keudell, W. Jacob, J. Appl. Phys. 86, 3988 (1999).

[14] S. Cernusca, H.P. Winter, F. Aumayr, R. Díez Muiňo, J.I. Juaristi, Nucl. Instr. Meth. in Phys. Res. B 203, 1 (2003).

[15] H. Zhang, F.W. Meyer, H.M. Meyer III, M.J. Lance, Vacuum 82, 1285 (2008).

[16] F.W. Meyer, H. Zhang, M.J. Lance, H.F. Krause, Vacuum 82, 880 (2008). 